\title{
|ESPECIFICIDADES DE USUÁRIOS(AS) DE DROGAS VISANDO UMA ASSISTÊNCIA BASEADA NA HETEROGENEIDADE
}

\author{
Drug Users' Specifics Aiming the Assistance \\ Based in the Heterogeneity \\ Especificidades de Usuários (as) de Drogas \\ Visando una Asistencia Basada en la Heterogeneidad
}

Jeane Freitas de Oliveira ${ }^{1}$

Enilda Rosendo do Nascimento

Mirian Santos Paiva ${ }^{3}$

\begin{abstract}
Resumo
0 artigo enfoca a heterogeneidade de grupos e de pessoas usuárias de drogas, com ênfase nas especificidades de mulheres. Trata-se de revisão da literatura sobre o fenômeno das drogas e saúde, a partir de textos selecionados que utilizam a variável empírica sexo. Foram identificadas diferenças no consumo de drogas entre homens e mulheres e especificidades entre mulheres tanto em relação a aspectos epidemiológicos quanto aos determinantes sócio-culturais do fenômeno. Identificou-se que a taxa de consumo, tipo da droga, idade, mortalidade e comorbidade foram aspectos responsáveis pelas principais diferenças de gênero. Especificamente em grupos de mulheres, foram identificadas diferenças relacionadas ao tipo de droga versus idade e procura por tratamento versus tipo de atividade desenvolvida pela unidade de saúde. Os dados demonstram que a assistência às pessoas usuárias de drogas deve contemplar especificidades individuais e de grupos.
\end{abstract}

Palavras-chave: Drogas llícitas. Identidade de Gênero. Saúde da Mulher.

\begin{abstract}
This paper deals with the issue of heterogeneity in drug addicted groups/people, emphasizing women's specifics. A concise survey of the literature on drugs phenomenon and health of works reporting the use of gender as an empiric variable is presented. Differences were found in drug consumption habits between men and women. Moreover, women's habits were shown to be influenced by specifics regarding both epidemiologic aspects and socio cultural elements. The main gender differences were observed on consumption rate, kind of drug, age, mortality and comorbidity. Concerning women groups, the main differences were found relating both kind of drug versus age and treatment seek versus kind of activity developed in the health unit. Examination of the data leads to the conclusion that assistance to drug users should take specifics of groups/people into account.
\end{abstract}

Keywords:

Street drugs. Gender Indentity. Women's Health.

\section{Resumen}

El artículo enfoca la heterogeneidad de grupos y de personas usuarias de drogas, con énfase en las especificidades de mujeres. Se trata de una revisión de la literatura sobre el fenómeno de las drogas y la salud, a partir de textos seleccionados que utilizan la variable empírica sexo. Fueron identificadas diferencias en el consumo de drogas entre hombres y mujeres y especificidades entre mujeres, tanto en relação a aspectos epidemiológicos, cuanto a las determinantes socioculturales del fenómeno. Se identificó que la tasa de consumo, tipo de droga, edad, mortalidad y comorbidad fueron aspectos responsables por las principales diferencias de género. Específicamente en grupos de mujeres, fueron identificadas diferencias relacionadas al tipo de droga versus edad y busca por tratamiento versus tipo de actividad desarrollada por la unidad de salud. Los datos demuestran que la asistencia a las personas usuarias de drogas debe contemplar especificidades individuáis y de grupos.

Palabras clave:

Drogas llícitas. Identidad de Género. Salud de la Mujer.

'Doutoranda do ISC/UFBA; professora da Escola de Enfermagem/UFBA, membro do Grupo de Estudos sobre saúde da Mulher - GEM/EEUFBA, bolsista da FAPESB; ${ }^{2}$ Prof. Dra. da Escola de Enfermagem da UFBA, componente do GEM/EEUFBA, coordenadora da Pós-graduação da EEUFBA; ${ }^{3}$ Profa. Dra. da Escola de Enfermagem da UFBA, membro do GEM/UFBA, coodenadora da linha de pesquisa Mulher,gênero e saude. 


\section{INTRODUÇÃO}

Atualmente, o consumo de drogas é considerado um problema social e de saúde pública de ordem mundial ${ }^{1}$. Os impactos sociais e de saúde causados por este problema apresentam-se distintos para cada sociedade a depender, dentre outros aspectos, das representações e significados atribuídos ao uso de drogas e, em conseqüência, às pessoas usuárias. Além disso, devem-se levar em consideração questões de ordem individual e dos grupos no interior de uma mesma sociedade.

Embora a heterogeneidade social e cultural das(os) usuárias(os) de drogas seja consenso na literatura ${ }^{2-4}$, ainda há uma "tendência à homogeneização, como se todos pertencessem a uma mesma categoria social e devessem ser vistos a partir de um mesmo enfoque" 5 . Essa idéia pode mascarar a necessidade de ampliar o leque de atividades assistenciais de cunho preventivo, educativo e de tratamento com vistas a contemplar os distintos grupos de usuários, assim como dificultar a análise mais aprofundada de pesquisas.

Nesse sentido, reconhece-se que as mulheres dependentes de substâncias psicoativas constituem um subgrupo diferenciado, com características e necessidades próprias, tanto para diagnóstico como para tratamento. Até recentemente, o uso dessas substâncias era considerado um problema do mundo masculino ${ }^{6}$, sendo as mulheres sub-representadas em estudos sobre esta temática. Do mesmo modo, o planejamento e a implantação de intervenções para pessoas usuárias de drogas estão baseados em necessidades masculinas, com pouca consideração para quaisquer diferenças entre os sexos, sejam elas fisiológicas, psicológicas ou sociais ${ }^{7}$. As pesquisas sobre 0 consumo de drogas por mulheres, datadas do início da década de 1980, em sua maioria, estão centradas no álcool e no tabaco, enfatizam aspectos farmacológicos e efeitos negativos sobre o organismo com destaque para as repercussões à saúde reprodutiva, apresentam uma abordagem condenatória ${ }^{8}$.

A incorporação da categoria gênero em pesquisas sobre - uso de substâncias psicoativas possibilita 0 reconhecimento do impacto sócio-cultural em construções da masculinidade/feminilidade sobre o uso individual e em grupo ${ }^{9}$, demarcando novas perspectivas cognitivas em relação às orientações conceituais e metodológicas dos agentes públicos responsáveis pelo enfrentamento das demandas sociais, traduzíveis em políticas públicas ${ }^{10}$.

A implantação e implementação de ações que conduzam a uma assistência equânime devem estar respaldadas, sobretudo, no reconhecimento das diferenças de gênero, pois existem evidências de que a questão do consumo de drogas é permeada por relações de poder determinando modos diferentes de acesso e consumo entre mulheres e homens.

Neste sentido, considera-se gênero "um sistema de signos e símbolos que denota relações de poder e hierarquia entre os sexos e modos diferentes de expressão no interior de relações do mesmo sexo" 11 , portanto, "algo relacional e transversal, interativo com classe social, raça, diferenças de geração, cultura, e não uma condição que determina, por si só, diferenciais de vulnerabilidade"12.
Este artigo tem o propósito de discutir heterogeneidade de pessoas usuárias de drogas a partir de dados publicados no período de 1995 a 2005 em textos de artigos e livros cientíicos relativos à problemática das drogas.

\section{METODOLOGIA}

Trata-se de uma revisão de literatura relacionada com a problemática das drogas que teve como objeto de estudo a heterogeneidade de pessoas usuárias de drogas. As informaç̃oes apresentadas foram produzidas a partir da identificação e seleção de textos publicados em livros e periódicos no período de dez anos, tendo como recorte temporal os anos compreendidos entre 1995 e 2005. Para tanto, dividiuse a consulta em duas partes, a primeira, englobando os periódicos indexados e constantes nas bases de dados Medline, LILACS e Scielo, e a segunda, os livros que integram o acervo das bibliotecas da Escola de Enfermagem, do Instituto de Saúde Coletiva e do Centro de Estudos e Terapia ao Abuso de Drogas - CETAD, instituições pertencentes à UFBA. Para ambas as consultas foram usados como descritores: drogas, substâncias psicoativas, consumo de drogas e mulher, gênero, sexo.

As buscas se desenvolveram nos meses de junho/2005 a janeiro/2006 incluindo, além dos textos (artigos e livros) em português, aqueles de língua inglesa e espanhola. Neste processo, considerados o objeto e o objetivo do estudo, foram excluídos os textos que não tratavam da heterogeneidade das pessoas usuárias de drogas e os que versavam sobre drogas medicamentosas.

De acordo com o objetivo proposto, foram selecionados e utilizados 39 textos que abordavam questões relacionadas ao fenômeno das drogas versus sexo/gênero. Os dados foram organizados qualitativamente, seguindo as etapas da análise de conteúdo temática proposta por Bardin ${ }^{13}$, com a finalidade de categorizar e articular os achados para melhor compreendêlos. A análise foi feita sob a perspectiva de gênero, sendo identificados dois principais temas: a) diferenças entre homens e mulheres usuários(as) de drogas; b) especificidades de mulheres e de grupos de mulheres usuárias de drogas.

0 primeiro tema está relacionado às diferenças encontradas entre mulheres e homens quanto: taxas de consumo, tipos de drogas, iniciação e manutenção do consumo, motivação para o consumo, situações de violência enfrentadas e padrões de relações sociais.

0 segundo refere-se às características da população feminina usuária de drogas com destaque para barreiras enfrentadas na busca e permanência de tratamento do uso de drogas, idade, drogas utilizadas e agravos à saúde. Neste tema estão inclusas, também, necessidades e particularidades de grupos de mulheres em uso de drogas em distintos contextos sócio-culturais.

\section{RESULTADOS E DISCUSSÕES}

\section{Diferenças entre homens e mulheres usuários(as) de drogas}

Estudos epidemiológicos com populações específicas ou com a população em geral em diferentes países têm mostrado que a taxa de consumo de drogas é mais elevada entre os homens. Entretanto, vem sendo registrada a diminuição da 
proporção entre homens e mulheres para as drogas de um modo geral e a predominância do uso de medicamentos, mais especificamente benzodiazepínicos, estimulantes e orexígenos, pela população feminina ${ }^{14-17}$. Não obstante, o uso dessas substâncias entre adolescentes do sexo feminino, em todo mundo, está aumentando, e, nos Estados Unidos, elas superam os meninos no uso de estimulantes e tranqüilizantes ${ }^{6}$, tornando-se mais vulneráveis, quando adultas, a desenvolverem quadro de dependência ${ }^{18}$.

Em relação ao tipo de drogas consumido, os homens, em sua maioria, estão mais propensos a usar drogas ilícitas mais precocemente, por mais tempo, em quantidade e com uma freqüência muito maior que as mulheres ${ }^{19}$. Entretanto, em algumas comunidades periféricas de Salvador, Bahia, o consumo de crackpor mulheres, especialmente aquelas que se dedicam ao trabalho com o sexo, vem se expandindo, superando as taxas apresentadas pelos homens. 0 fácil acesso e o baixo valor da droga, a aceitação do sexo como moeda de troca e a preferência de homens que fazem uso de drogas utilizarem aquelas consideradas como menos danosas foram apontados como fatores facilitadores para este quadro ${ }^{20}$.

Mulheres que consomem álcool e outras drogas tornam-se mais vulneráveis a determinados danos e agravos pessoais e à saúde, dentre eles o risco de contrair infecção pelo HIV, seja por questões de ordem fisiológica - metabolismo menos tolerante aos efeitos das drogas - seja por construções sócioculturais sobre a feminilidade, conferindo aos homens maior poder de decisão nas relações heterossexuais ${ }^{3,19,21}$. A representação de que o consumo de drogas é um comportamento desviante ${ }^{22}$ e de que a mulher que adota tal conduta está duplamente contrariando as normas sociais, diante da possibilidade de não cumprir os papéis sociais e culturais a elas destinados, quais sejam: mãe, esposa e cuidadora da família, contribui para que as mulheres façam um consumo às escondidas, sendo consideradas como uma "fração oculta da sociedade" .

As diferenças fisiológicas entre homens e mulheres determinam distintos agravos de saúde para ambos, com desigualdades nas taxas de mortalidade e comorbidade. Para as mulheres, os problemas de saúde decorrentes do consumo de drogas além de incidirem diretamente em seus corpos, poderão afetar também o feto, quando estas engravidam. Alterações no ciclo menstrual, na fertilidade, na gestação, no parto, no risco de contrair e de desenvolver doenças sexualmente transmissíveis são freqüentemente registradas entre mulheres usuárias ${ }^{23}$. A comorbidade relativa aos transtornos depressivos e à ansiedade apresenta-se mais freqüentemente entre mulheres, enquanto os homens tendem a desenvolver comorbidades relacionadas aos transtornos de personalidade ${ }^{24}$.

A heterogeneidade entre usuárias(os) de drogas também se manifesta nas relações sociais, especialmente quando a droga de abuso é considerada social e juridicamente ilícita. Estando nesta situação, as mulheres tendem a manter relacionamentos com homens usuário de drogas, enquanto estes preferem estar com as mulheres não usuárias ${ }^{3}$.

Quanto às formas utilizadas para aquisição de drogas, embora a literatura aponte que em geral as mulheres tendam a valer-se do corpo como moeda de troca e os homens envolvamse mais comumente com o narcotráfico, já vem sendo registrado, de forma ainda discreta, o envolvimento de mulheres em ações nesse mercado, havendo destaque para as chamadas "mulas" e até mesmo a chefia de pontos de vendas ${ }^{25}$.

\section{Especificidades de mulheres e de grupos de mulheres usuárias de drogas}

As mulheres usuárias de drogas enfrentam barreiras de ordem estrutural; sistêmica; social, cultural e pessoal na busca e permanência de tratamento. As barreiras sistêmicas refletem a falta de habilidade para identificação da diferença de gênero, tanto por parte de pesquisadoras(es) e formuladores de política em saúde, como por parte da população feminina, para influenciar as políticas e decisões sobre alocação de recursos para programas que respondam a necessidades especificas de mulheres.

As barreiras estruturais dizem respeito às práticas e políticas adotadas pelos programas e serviços de assistência à saúde e estão relacionadas com as abordagens utilizadas, localização do serviço e os custos para acessá-lo, rigidez na programação e nos critérios de admissão, entre outros. As barreiras de ordem social, cultural e pessoal fazem referências aos comportamentos e papéis predeterminados social e culturalmente para as mulheres ${ }^{26}$.

Diferença significativa foi registrada na freqüência de mulheres usuárias de drogas e mulheres parceiras e/ou acompanhantes e familiares de usuários de drogas em um serviço de saúde especializado no tratamento do uso de drogas, em Salvador, Bahia, que desenvolve atividades assistenciais internas e externas à unidade de saúde. Enquanto nas atividades internas as mulheres atendidas se apresentavam como acompanhantes, familiares e/ou parceiras de usuários, nas atividades externas houve registro significativo de atendimento de mulheres usuárias de drogas. Fica evidente a importância da diversificação de abordagens do tema de modo a incluir os distintos grupos de mulheres envolvidas com o uso de drogas nos mais diversos contextos ${ }^{20}$.

A idade e o tipo de droga também são apontados como fatores demarcadores de diferenças entre mulheres usuárias de drogas. As pesquisas, de um modo geral, apontam que mulheres jovens, além de consumir álcool, fazem uso de outras drogas como maconha, crack e cocaína, enquanto mulheres adultas e idosas fazem uso, mais freqüentemente, de tabaco, álcool e medicamentos, especialmente os tranqüilizantes. Já os medicamentos inibidores do apetite têm uso predominante por mulheres jovens e adultas na busca de atender aos padrões de beleza determinados socialmente ${ }^{15,16}$.

Com relação a grupos específicos de mulheres em uso de substâncias psicoativas, estudos realizados nos Estados Unidos identificaram consumo e necessidades específicas para mulheres grávidas, mulheres responsáveis por crianças, trabalhadoras do sexo, presidiárias e pertencentes a minorias raciais e étnicas. Para estes grupos, os serviços especializados, de transporte, de cuidados destinados às crianças e para grupos minoritários foram apontados como necessidades específicas ${ }^{21}$. Considerando a realidade brasileira em relação à assistência à 
pessoa usuária de drogas, pode-se dizer que estas necessidades, também, devem ser ponderadas para mulheres usuárias de drogas vinculadas aos grupos de baixo capital sócio-cultural.

Em Salvador, Bahia, foram identificados três grupos específicos de mulheres usuárias de drogas que diferem pelo tipo de droga utilizado e pelas formas de aquisição, cujas características estão relacionadas a papéis e atividades sociais e culturalmente direcionadas às mulheres ${ }^{20}$. Um dos grupos era composto por donas-de-casa de diversas faixas etárias com uso preferencial por maconha e álcool em espaço privado e vinculado ao parceiro, o qual, na maioria das vezes é também o fornecedor das substâncias utilizadas. Por manterem atividades atreladas ao papel de mãe e dona de casa (cuidar da casa, dos filhos, do esposo) e consumir drogas ocultamente, elas são de alguma forma respeitadas, não enfrentando, assim, muitas situações de discriminação.

Mulheres adultas jovens que desempenham atividades como profissionais do sexo e que fazem consumo concomitante de álcool, maconha, crack e cocaína formam outro grupo. As adversidades enfrentadas no trabalho que executam são apontadas como motivos para o consumo de drogas, as quais são adquiridas com recurso oriundo do seu trabalho. Muitas são chefes de família, sustentando filhos, pais idosos e outros parentes, sendo assim mais discriminadas pela atividade que exercem do que pelo uso de drogas.

0 terceiro grupo, denominado "piriguetes", era composto por mulheres jovens, moradoras de outras comunidades, que não têm um papel definido dentro da comunidade e que se sujeitam a qualquer coisa para ter acesso às drogas, principalmente manter relações sexuais com um ou mais usuários de drogas, sem uso de preservativo. Este grupo é considerado uma ameaça para as outras mulheres da comunidade pela possibilidade de envolvimento com seus parceiros e/ou clientes com risco de transmissão de doenças sexualmente transmissíveis, pela prática do sexo não seguro. Assim, essas mulheres estão mais sujeitas às situações de violência e discriminação que as demais, tanto pelos homens como pelas próprias mulheres ${ }^{20}$.

Pouco se sabe sobre agravos à saúde em grupos específicos de usuárias de drogas. Por exemplo, qual o impacto para a saúde do consumo de drogas por mulheres no sistema presidiário ${ }^{9}$, por mulheres que adotam o sexo casual e a prostituição como estratégias para obtenção de drogas e, ainda, por aquelas que mantêm relacionamentos sexuais com homens usuários de drogas, sem adoção de medidas preventivas? Parceiras de usuários de drogas que não fazem uso de drogas também estão sujeitas a estes agravos? ${ }^{6}$ Por fim, o que dizer da vulnerabilidade de mulheres que fazem sexo com outras mulheres usuárias de drogas?

\section{CONCLUSÃO}

A literatura pertinente aponta a taxa de consumo, o tipo da droga, a idade, taxas de mortalidade e comorbidade como principais diferenças de gênero em relação ao uso de drogas.

De fato, observou-se que a taxa de consumo de drogas é mais elevada entre os homens, embora venha diminuindo a proporção entre os sexos; há registros de predominância do uso de medicamentos, mais especificamente benzodiazepínicos, estimulantes e orexígenos, pela população feminina, embora, em algumas comunidades com características próprias, o consumo de crack por mulheres, especialmente aquelas que se dedicam ao trabalho com o sexo, venha se expandindo, superando as taxas apresentadas pelos homens. Em relação à idade das(os) consumidoras(es), observa-se elevação do uso dessas substâncias entre adolescentes do sexo feminino, chegando a superar a taxa de uso masculino em certos países.

Desigualdades nas taxas de mortalidade e comorbidade foram identificadas no tocante à morbimortalidade. Para as mulheres, foram registradas alterações no ciclo menstrual, na fertilidade, na gestação, no parto, no maior risco de contrair e desenvolver doenças sexualmente transmissíveis; e há predominância dos transtornos depressivos e ansiedade, enquanto os homens tendem a desenvolver transtornos de personalidade.

Quanto às especificidades da manifestação do fenômeno do consumo de drogas no interior do grupo de mulheres, foram identificadas diferenças em relação ao tipo de droga e idade, procura por tratamento e tipo de atividade desenvolvida pela unidade de saúde. Desse modo, foi registrada diferença significativa na freqüência ao serviço de saúde por mulheres usuárias de drogas e acompanhantes. As primeiras preferiam o atendimento em atividades externas aos serviços de saúde, enquanto nas atividades intramuros, as mulheres registram-se como acompanhantes.

As mulheres jovens, além do consumir álcool fazem uso de outras drogas como maconha, crack e cocaína, enquanto mulheres adultas e idosas fazem uso, mais freqüentemente, de tabaco, álcool e medicamentos, especialmente os tranqüilizantes. Já os medicamentos inibidores do apetite têm uso predominante por mulheres jovens e adultas na buscam de atender a padrões de beleza determinados socialmente.

Finalmente, foram identificadas diferenças no tipo de droga utilizado e as formas de sua aquisição entre mulheres que podem estar referidas aos papéis culturalmente atribuídos às mulheres.

Os dados apresentados refletem a necessidade de implantação e implementação de estratégias para 0 enfrentamento do fenômeno das drogas baseadas em especificidades individuais e de grupos de forma a contemplar a heterogeneidade dos sujeitos em seus distintos contextos. Para tanto, torna-se imperativo estimular a ampliação e/ou incorporação de questões relacionadas ao consumo de drogas nos currículos dos inúmeros profissionais de saúde e a realização de mais estudos, em todas as regiões do mundo, envolvendo homens e mulheres em seus distintos grupos e contextos sócioculturais, numa perspectiva de gênero. 


\section{Referências}

1. Organização das Nações Unidas- ONU. Programa para o Controle Internacional de Drogas. Escritório das Nações Unidas Contra Drogas e Crime da ONU- UNODC. [on line]. Brasilia (DF); 2005 [citado 20 mar 2006]. Disponível em: http://www.unodc.org.br.

2. Spricigo IS, et al. Atenção ao usuário de drogas: um espaço para o enfermeiro. Texto\& Contexto Enferm 2004 abr/jun; 13(2): 296-302.

3. Ferreira $P$, et al. Percebendo as facilidades e dificuldades na implantação de serviços abertos em álcool e drogas. Texto\&Contexto Enferm. 2004 abr/jun; 13(2): 209-16.

4. Organização Mundial de Saúde/Organização Panamericana de Saúde-OMS/OPAS. Declaração de Caracas. Conferência Regional para Reestruturação da Atenção Psiquiátrica na América Latina no contexto dos Sistemas Locais de Saúde-SILOS; 1990 nov 14 ; Caracas; 1990.

5. Ministério da Saúde (BR). Secretaria de Atenção à Saúde. SVC/CN/ DST/AIDS. A política do Ministério da Saúde para a atenção integral a usuários de álcool e outras drogas. Brasília (DF); 2004.

6. GelbckeFL, Padilha MICS. Ofenômeno das drogas no contesto da promoção da saúde. Texto\&Contexto Enferm 2004 abr/jun; 13(2): 272-79.

7. Polit DF, Hungler BP. Fundamentos da pesquisa em enfermagem. Porto Alegre(RS): Artes Médicas; 1995.

8. Minayo MCS. 0 desafio do conhecimento: pesquisa qualitativa em saúde. São Paulo (SP): Hucitec; 1999.
9. Friedrich DBC, Sena RR.Um novo olhar sobre o cuidado no trabalho da enfermeira em unidades básicas de saúde em Juiz de Fora-MG. Rev Latino-am Enfermagem 2002 nov/dez; 10(6).

10. Filizola CLA, et al. Compreendendo o alcoolismo na família. Esc Anna Nery Rev Enferm 2006 dez; 10 (4): 660-70.

11. Cartana MHF, Santos SMA, Fenili RM, Spríigo IS. Prevenção do uso de substâncias psicoativas. Texto\&Contexto Enferm 2004 abr/jun; 13(2): 286-89.

12. Melles AM, Zago MMF. Análise da educação de clientes/pacientes na literatura brasileira de enfermagem. Rev Latino-am Enfermagem 1999; 7(5): 85-94.

13. Oliveira AGB, Alessi NP. 0 trabalho de enfermagem em saúde mental: contradições e potencialidades atuais. Rev Latino-am Enfermagem 2003 maio/jun; 11(3): 333-40.

14. Fontanella BJB, Turato ER. Barreiras na relação clínico paciente em dependentes de substâncias psicoativas procurando tratamento. Rev Saude Publica 2002 ago; 36(4): 439-78.

\section{Agradecimentos}

À FAPESB - Fundação de Amparo à Pesquisa do Estado da Bahia pelo apoio financeiro na modalidade de bolsa de doutorado para o desenvolvimento da pesquisa que originou este artigo; a Henrique Celso Santos, pelas significativas contribuições à construção deste trabalho. 